\title{
Prognostic factors for olfactory groove meningioma with nasal cavity extension
}

\author{
Ji Zhang ${ }^{1, *}$, Ke Sai ${ }^{1, *}$, Zheng-Quan Zhu ${ }^{2, *}$, Fu Hua Linn ${ }^{1, *}$, Zi-Feng Wang ${ }^{3, *}$, Yong-Ming \\ Chen $^{4, *}$, Chun-Yu Huang ${ }^{5, *}$, Yun-Lin Ye ${ }^{6, *}$, Xiao-Li Wang ${ }^{7, *}$, You-Ping Li ${ }^{8, *}$, Shu-Xin \\ Sun $^{1, *}$, Wei-Ying Zhong ${ }^{9, *}$, Jian-Bin Chen ${ }^{10, *}$ and Yun-Qiang Yang ${ }^{11}$ \\ ${ }^{1}$ Department of Neurosurgery, State Key Laboratory of Oncology in South China, Sun Yat-sen University Cancer Center, \\ Collaborative Innovation Center for Cancer Medicine, Guangzhou, China \\ ${ }^{2}$ Department of Neurosurgery, Tumor Hospital Affiliated of Xinjiang Medical University, Ürümqi, China \\ ${ }^{3}$ State Key Laboratory of Oncology in South China, The Cancer Center of Sun Yat-sen University, Collaborative Innovation \\ Center of Oncology, Guangzhou, China \\ ${ }^{4}$ Department of Gastric Surgery, State Key Laboratory of Oncology in South China, Sun Yat-sen University Cancer Center, \\ Collaborative Innovation Center for Cancer Medicine, Guangzhou, China \\ ${ }^{5}$ Department of Endoscopy, State Key Laboratory of Oncology in South China, Sun Yat-sen University Cancer Center, \\ Collaborative Innovation Center for Cancer Medicine, Guangzhou, China \\ ${ }^{6}$ Department of Urology, State Key Laboratory of Oncology in South China, Sun Yat-sen University Cancer Center, \\ Collaborative Innovation Center for Cancer Medicine, Guangzhou, China \\ ${ }^{7}$ Department of General Surgery, Shang Jin Nan Fu Hospital, West China Hospital, Sichuan University, Chengdu, Sichuan, \\ China \\ ${ }^{8}$ Department of Neurosurgery, The First Affiliated Hospital of Nanchang University, Nanchang, Jiangxi, China \\ ${ }^{9}$ Department of Neurosurgery, Qilu Hospital, Shandong University, Jinan, China \\ ${ }^{10}$ Department of Neurosurgery, Tongji hospital, Tongji Medical College, Huazhong University of Science and Technology, \\ Wuhan, China \\ ${ }^{11}$ Department of Oral and Maxillofacial Surgery, Tongji Hospital, Tongji Medical College, Huazhong University of Science and \\ Technology, Wuhan, China \\ *These authors contributed equally to this work
}

Correspondence to: Yun-Qiang Yang, email: yangyun720@163.com

Keywords: olfactory groove meningioma; communicating; recurrence; prognosis factors

Received: August 06, $2017 \quad$ Accepted: November 28, $2017 \quad$ Published: December 19, 2017

Copyright: Zhang et al. This is an open-access article distributed under the terms of the Creative Commons Attribution License 3.0 (CC BY 3.0), which permits unrestricted use, distribution, and reproduction in any medium, provided the original author and source are credited.

\section{ABSTRACT}

Objectives: Meningioma recurrence remains a significant issue. No study has described the relationship between the clinical features and prognosis of communicating meningioma that primarily originates from the olfactory groove. The aim of the study was to identify prognostic factors of communicating olfactory groove meningiomas that could be stratified according to their risk of recurrence.

Results: A Simpson grade one or two resection was achieved. Complications with cerebrospinal rhinorrhoea occurred in two patients: one required reoperation, and the other was managed successfully with external drainage of lumbar cistern. There were 5 known clinical recurrences within the median follow-up of more than 5 years. The median 5 -year recurrence-free survival for patients was $\mathbf{8 8 . 4 \%}$. Factors such as gender, tumour size, T2 signal and the hyperostotic bone had no significant effect on recurrence-free survival. However, recurrence was activated by oedema range, hyperostosis, dural tail sign and tumor texture $(p<0.05)$. Interestingly, female patients with the disease were younger than males at diagnosis, and the difference was statistically significant $(p=0.013)$. 
Conclusions: Based on these features of communicating olfactory groove meningiomas, different strategies may be adopted for the follow-up and subsequent treatment. Due to the relatively uncommon incidence, more investigations into the clinical behaviour of this entity are crucial.

Patients and Methods: A retrospective study of $\mathbf{4 3}$ patients harbouring olfactory groove meningiomas invading the ethmoid or nasal cavity was conducted at three medical centers from 2000 to 2010. The records were reviewed for clinical presentations, imaging studies, surgical observation, histological features and follow-up.

\section{INTRODUCTION}

Meningiomas are commonly considered to grow slowly, and cured by conventional surgery [1]. However, recurrence is often unavoidable due to incomplete tumour resection or subtotal resection [2]. The clinical behaviour of meningiomas originating from olfactory groove has been documented, but very little is known regarding the prognostic factors of olfactory groove meningiomas (OGMs) with extension into the ethmoid sinuses or nasal cavity [3]. The majority of the disease relapses occur among World Health Organization (WHO) grade I patients [4]. Preoperatively failing to understand the clinical behaviour of these tumours can cause inadequate attention for treatment and follow-up. Based on this information, there is an urgent need to identifying reliable prognosis factors of communicating OGM to adopt different treatments and an efficient follow-up strategy for individual patients.

\section{RESULTS}

No patient died of surgery. All patients received clinical examination and MRI assessments. Total resection of tumor by the three neurosurgeons was achieved in the investigated patients. Follow-up procedures were put into effect in accordance with a standard clinical protocol that consisted of MRI performed 3 months after surgery in the first year and every one or two years thereafter. Additional MRI studies were performed whenever clinical signs and/ or symptoms were noted and/or a relapse was suspected. At the time of study closing, five patients had recurrent tumours. Histologically, the majority of tumours were the meningothelial type (WHO Tumor classification criteria, 2007). Significant PTBE was associated with a longer hospital stay, difficulty of surgical resection, soft tumor texture, dural tail sign and a risk of intracranial hypertension compared with meningiomas with less oedema in our series.

In a detailed analysis of the factors increasing communicating OGM recurrence, five clinical features of the disease exhibited an adverse impact on participants' RFS, namely younger age based on gender $(P=0.026)$ (Table 1), presence of brain oedema range (bandwidth) $\geq 20 \mathrm{~mm}(P=.023)$, soft tumor texture $(p=0.037)$, hyperostosis $(P=0.037)$ and dural tail sign $(p=0.019)$ (Table 2). These factors, including gender $(P=0.129)$, size $(P=0.671), \mathrm{T} 2$ signal $(P=0.671)$ and bone hyperostosis $(P=0.598)$, were not able to predict RFS of OGM (Table 2). The overall actuarial disease-free survival for all patients in our series was $88.4 \%$ (Figure 1). Five patients developed local tumour relapse (Figure 2).

\section{DISCUSSION}

Olfactory groove meningiomas (OGM) arise from the weakest part of the skull base. The cribriform plate has an extension into the ethmoid or nasal cavity, which is an uncommon site of occurrence [5]. Tumours located in this area perhaps vary with respect to clinical characteristics compared with other regions. Forecasting OGM recurrence in a single case remains challenging $[1,2]$. This study identified potentially relevant clinical prognostic factors based on long-term follow-up to predict tumour relapse.

In the present study, we introduce OGM with extracranial extension as a clinical entity distinct from meningiomas with a predominant origin from the sphenoid wing, cavernous sinus, convexity, or petrous ridge. We believe that meningiomas that arise predominantly from olfactory groove with extracranial extension have important clinical implications. The most relevant clinical features have been systematically investigated in our surgical series. Males and females each accounted for $50 \%$ of the patients. The mean age at presentation was $47 \mathrm{yrs}$. Of interest, there was a statistically significant difference for the males compared with females in terms of age; females were, on average (44 yrs), younger than their male counterparts (50.2 yrs; $p=0.013$ ). We conclude that female patients are younger than their male counterparts at the time of diagnosis. A similar report about the relationship between age and gender in patients with communicating OGM was not searched in the literature. In contrast to our finding, the average age at presentation for women was older than men, which was statistically significant, but the authors did not explain this difference [6]. No significant differences were found with regard to patients' age and sex distribution among patients [7]. No recorded studies confirmed the prognostic value of patient age $[8,9]$. Several studies have demonstrated a worse outcome in younger patients $[10,11]$.

All patients in our series underwent surgical resection via bilateral or unilateral inferior frontal 
Table 1: Female patients are younger than males at communicating OGM diagnosis

\begin{tabular}{lccc}
\hline Age/Gender & Female & Male & $P$ value \\
\hline 45 & 10 & 3 & 0.026 \\
$>45$ & 12 & 18 & \\
\hline
\end{tabular}

Table 2: Clinical and radiographic characteristics of communicating OGM

\begin{tabular}{lccc}
\hline Variables & Patient distribution & No. of recurrences & P value \\
\hline Gender & 21 & 2 & 0.708 \\
$\quad$ Males & 22 & 3 & \\
$\quad$ Females & 7 & 3 & 0.023 \\
Oedema range & 36 & 2 & \\
$\quad \geq 20 \mathrm{~mm}$ & & & 0.833 \\
$\quad<20 \mathrm{~mm}$ & 7 & 1 & \\
Tumor size & 36 & 4 & 0.037 \\
$\quad \geq 50 \mathrm{~mm}$ & & 3 & \\
$\quad<50 \mathrm{~mm}$ & 8 & 2 & 0.019 \\
Tumor texture & 35 & 4 & \\
$\quad$ soft & & 1 & \\
$\quad$ hard & 12 & 3 & 0.037 \\
Dural tail sign & 31 & 3 & \\
$\quad$ Yes & & 2 & \\
$\quad$ No & 8 & & \\
Hyperostosis & 35 & & \\
$\quad$ Yes & & & \\
$\quad$ No & & & \\
\hline
\end{tabular}

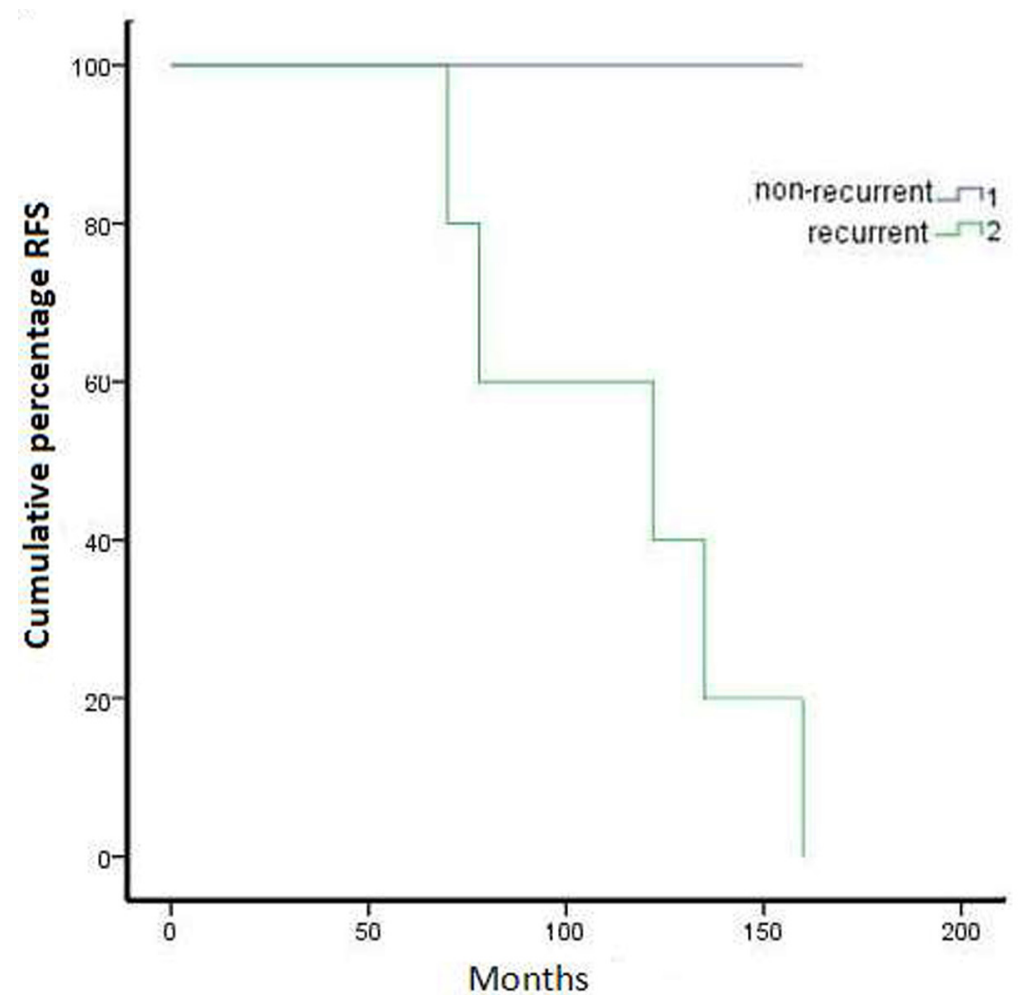

Figure 1: Kaplan-Meier representation of recurrence free survival over five years for all cases with communicating OGM. 
approach craniotomy, with or without assistance by nasal endoscopy. Complete removal of the OGM was attained in 67 to $100 \%$ of the examined cases according to different reports $[12,13]$. Macroscopically, the tumours were typically infiltrative into the bone of the nasal cavity or sinus. Hyperostosis was frequently macroscopically visible. Recurrence rate data of OGMs are controversial. Several reports have displayed a very low recurrence rate $[14,15]$. In contrast, some studies reported a high recurrence rate of up to $41 \%$ at the 10 -years follow-up in OGMs [13]. The tumour recurrence rate clearly relies on both the extent of tumour resection and the duration of the follow-up period, but these studies did not assess the relationship between tumour recurrence and clinical and pathological features.

Based on our results, 43 OGM cases with distinct prognoses were defined by a combination of clinical features that served as independent prognostic factor. In total, $11.6 \%$ of cases were classified with a bad prognosis indicative of a relapse within 5 years after tumour removal. Five high-risk factors involving obvious oedema ( $\geq 20 \mathrm{~mm})$, soft tumor texture, hyperostosis and dural tail sign, indicated a high frequency of recurrence. Some OGM cases with complete resection and greater oedema extension were also associated with a worse prognosis in our series $(p<0.05)$. The specific reasons for the association between oedema range and bad prognosis require further investigation. Our patients all exhibited peritumoural brain oedema (PTBE) on preoperative imaging, which is consistent with previous documents [16]. Oedema was associated with a shorter course of neurological symptoms [17]. Significant PTBE was associated with a longer hospital stay, difficulty of surgical resection, and a risk of intracranial hypertension compared with meningiomas with less oedema in our series. In our view, the easier the surgical plane is to detect, the easier the resection will be. When the arachnoid membrane interface was unclear, the resection was equally arduous. We noticed that patients without or less oedema were inclined to be those in whom the neurosurgeon could discern an explicit plane between the brain tissue and the tumour. This type of interface is frequently associated with PTBE and a disrupted arachnoid layer [18-20], which may explain why meningioma recurs, even following complete removal. Several causative factors for PTBE have been presented to date, including tumour location, size, histological differentiation, sex hormone receptors, venous stasis, vascularity, secretory activity, microcortical invasion, and macrophage infiltrates $[21,22]$. However, the relationship between PFS and oedema was not investigated in patients with communicating OGM patients.

To the best of our knowledge, this is the first series of communicating OGM cases reported to date in which clinical traits were systematically investigated with the recurrence of the disease. Oedema range, hyperostosis,

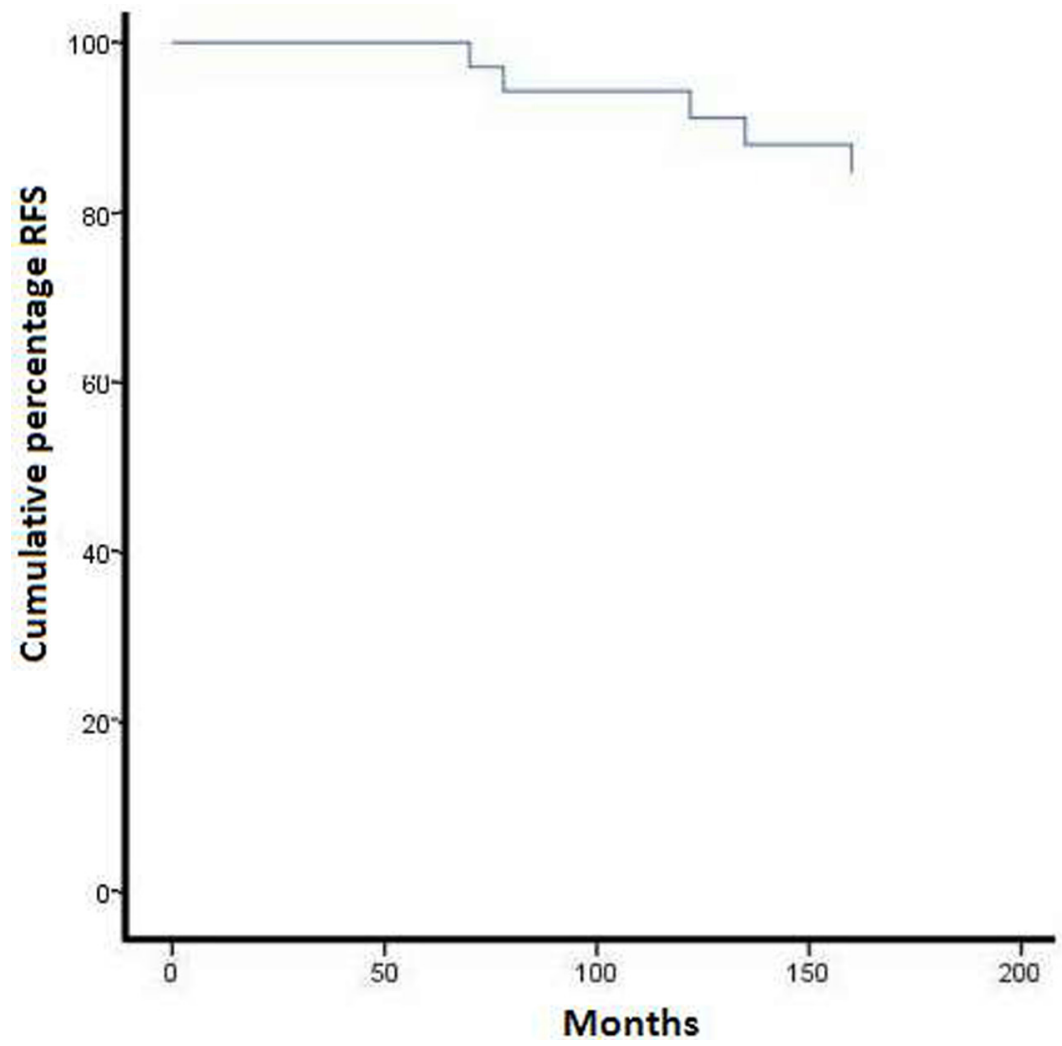

Figure 2: Survival in recurrent and non-recurrent cases. 
soft tumor texture, dural tail sign, and younger female patients with communicating OGM emerged as the relevant prognostic factors. Consequently, these 5 prognostic factors mentioned above were retained at the expense of a decreased frequency of cases. Altogether, these data indicate that the classification superinduces valuable prognostic information to that of the WHO grade alone and result in a more refined risk classification of communicating OGM.

No significant correlation was discovered among tumour sizes, simple gender, T2 signal and recurrence. In our surgical series, the overall tumour recurrence rate was $11.6 \%$ during a median follow-up period of 91.8 months. The 5-year locoregional disease control rate in the present series was $88.4 \%$, and the uncovered factors that were independently associated with locoregional recurrence included the involvement of oedema, hyperostosis and dural tail sign. Soft tumor texture equally reduced the RFS. A clustering of cases with bone hyperostosis is expected to be associated with relapse [22], and this finding was supported by our data.

In addition, these tumours clinically behave different from other positions of meningiomas given that grow rather large prior to presentation. By virtue of their subfrontal location, these patients present with nonspecific symptoms. In the light of their large size at diagnosis, it is not surprising that the morbidity of resecting these tumours is not trivial, with many of patients exhibiting obvious postoperative sequelae. We report the clinical characteristics of a group of patients presenting with OGM. Given the uncommon nature of these lesions, 43 consecutive patients were identified from three medical centers. Thus, the data definitively demonstrate a clinical course for these lesions that is truly different from other well-known meningioma classes (ie, cavernous sinus, sphenoid wing, etc). More investigations into the clinical behaviour of this entity, such as karyotype, are necessary before we can make meaningful conclusions about the clinical behaviour of these lesions [23].

\section{MATERIALS AND METHODS}

A total of 43 cases were retrospectively reviewed from three medical centers between 2000 and 2010. Data for these patients, including demographics, gender, radiographic material, pathology data, perioperative findings and postoperative evidence of recurrence on magnetic resonance imaging (MRI), were gathered by review of medical records. The most common presenting symptoms were olfactory impairment (16 patients), cognitive decline (11 patients), memory loss (9 patients), apathia (4 patients) and headache ( 3 patients). Onset of these symptoms is gradual, and they may not be noted early. These cases comprised 21 males and 22 females, aged 36 to 67 years (mean, $47.0 \mathrm{yrs}$ ).

We reviewed computed tomography (CT) and MR images to identify patients with tumours meeting our definition of OGM with extracranial invasion like (Figure 3A-3C). Tumours ranged from 4.0 to $8.0 \mathrm{~cm}$ in diameter (mean, $5.5 \mathrm{~cm}$ ). The tumours eroded the mucous membrane of the sinuses and extended into the sinal cavity. Age, gender, size, oedema, T2 signal, bone

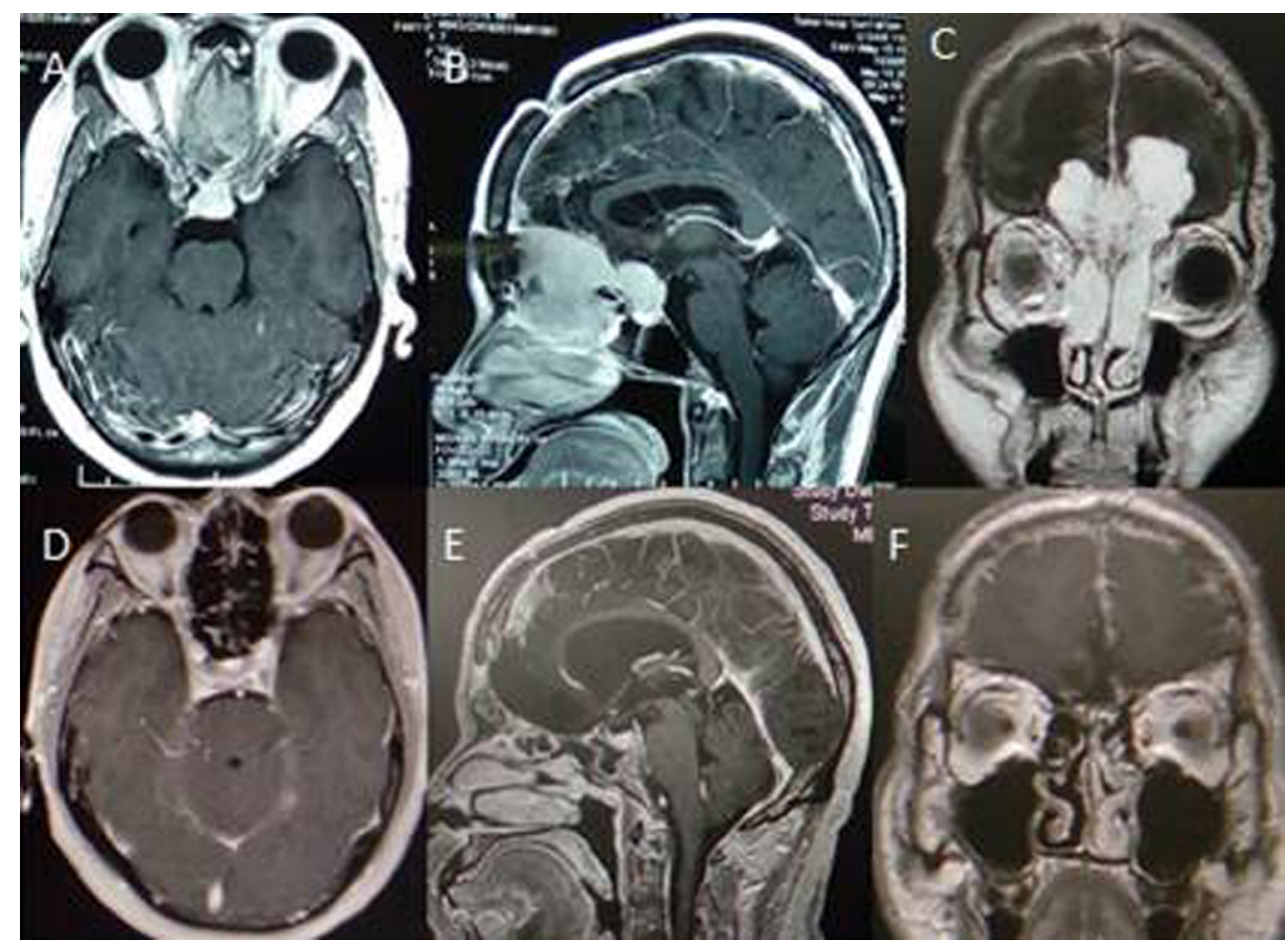

Figure 3: Cranial MR image displaying olfactory groove tumor with nasal cavity extension and follow-up with no recurrence. 
Table 3: Immunohistochemical panel of communicating OGM

\begin{tabular}{lcc}
\hline Antigen/antibody & Positive & Negative \\
\hline Epithelial membrane antigen (EMA) & 32 & 11 \\
Vimentin & 21 & 22 \\
Smooth muscle actin (SMA) & 16 & 27 \\
Progesterone receptor & 40 & 3 \\
Estrogen receptor & 0 & 43 \\
Epidermal growth factor receptor (EGFR) & 12 & 31 \\
\hline
\end{tabular}

hyperostosis and destruction were analysed in statistics. No adjuvant therapy was administered, with the exception of radiotherapy in two patients. Tumour histological diagnosis coincided with the WHO I criteria, with the exception of one atypical case. The classification of tumour pathology was diversified. All patients received follow-up with clinical examination and MRI studies three months and one year after surgery like Figure 3D-3F. Thereafter, patients were re-examined regularly at oneor two-year intervals based on each follow-up result. The duration of follow-up ranged from 66 to 159 months (median, 91.8 months).

\section{Surgical findings}

A bifrontal/unilateral subfrontal craniotomy with/ without endoscopic endonasal assistance was performed, providing wide access to the anterior fossa with minimal frontal lobe retraction while still enabling total tumour removal with a low morbidity rate and no mortality. As shown by preoperative MRI and confirmed intraoperatively, dural thickness around the base of tumour was disclosed in 16 cases; skull base hyperostosis and bone destruction at the tumour attachment area were observed in four and three cases, respectively. Bilateral extension of the tumour into the ethmoidal cells was observed on preoperative coronal and sagittal MRI in 22 cases, and unilateral extension was observed in 21 cases during surgery. Almost all lesions, regardless of the type, demonstrated an infiltrative growth pattern, frequently invading adjacent tissue.

\section{Pathologic features}

Calcifications and fragments of bone were macroscopically visible. Histological diagnosis was performed according to the WHO criteria. The pathologic classification of these neoplasms included meningothelial, transitional, fibrous, and psammomatous meningioma. Epithelial membrane antigen was positive in two-thirds of cases, although they were often weakly and focally observed. Rare lesions exhibited focal immunoreactivity with keratin, CD34, and S-100 protein. All cases were positive for proliferating cell nuclear antigen (PCNA) to a variable degree and intensity. Immunoreactivity was noted for progesterone receptors in all patients more frequently than for oestrogen receptors. Reactivity for smooth muscle actin was observed. The table provides a summary of the immunohistochemistry data obtained in our 43 patients (Table 3).

\section{Statistical analysis}

To establish the statistical significance of differences observed between two variables, the chi-square test was used for qualitative variables. A $P$-value was calculated for each comparison using two-tailed analysis, with significance assumed at the 0.05 level. Recurrence-free survival (RFS) curves were plotted according to the method of Kaplan-Meier method.

\section{Abbreviations}

OGM: Olfactory groove meningioma; WHO: World Health Organization; RFS: Recurrence-free survival; CT: Computed tomography; MRI: Magnetic resonance imaging; PTBE: Peritumoral brain edema.

\section{Author contributions}

J Zhang, K Sai, ZQ Zhu, FH Lin, ZF Wang, YM Chen, CY Huang and YL Ye, participated in the design of the study, performed the statistical analysis and collection of data, and drafted the manuscript. XL Wang, YP Li, SX Sun, WY Zhong and JB Chen recruited patients to the study, collected and organized the data. YQ Yang conceived of the study, revised and approved the final manuscript. All authors read and approved the final manuscript.

\section{ACKNOWLEDGMENTS}

The authors thank the editors and reviewers for their constructive comments.

\section{CONFLICTS OF INTEREST}

The authors declare that we have no competing interests. 


\section{FUNDING}

The study was supported by National Natural Science Foundation of China (No. 81500830).

\section{REFERENCES}

1. Saraf S, McCarthy BJ, Villano JL. Update on meningiomas. Oncologist. 2011; 16:1604-13. https://doi.org/10.1634/ theoncologist.2011-0193.

2. Yew A, Trang A, Nagasawa DT, Spasic M, Choy W, Garcia HM, Yang I. Chromosomal alterations, prognostic factors, and targeted molecular therapies for malignant meningiomas. J Clin Neurosci. 2013; 20:17-22. https://doi. org/10.1016/j.jocn.2012.02.007.

3. Aoyama H, Shirato H, Tago M, Nakagawa K, Toyoda T, Hatano K, Kenjyo M, Oya N, Hirota S, Shioura H, Kunieda E, Inomata T, Hayakawa K, et al. Stereotactic radiosurgery plus whole-brain radiation therapy vs stereotactic radiosurgery alone for treatment of brain metastases: a randomized controlled trial. JAMA. 2006; 295:2483-91. https://doi.org/10.1001/jama.295.21.2483.

4. Maillo A, Orfao A, Espinosa AB, Sayagues JM, Merino M, Sousa P, Lara M, Tabernero MD. Early recurrences in histologically benign/grade I meningiomas are associated with large tumors and coexistence of monosomy 14 and $\operatorname{del}(1 \mathrm{p} 36)$ in the ancestral tumor cell clone. Neuro Oncol. 2007; 9:438-46. https://doi. org/10.1215/15228517-2007-026.

5. Ildan F, Erman T, Gocer AI, Tuna M, Bagdatoglu H, Cetinalp E, Burgut R. Predicting the probability of meningioma recurrence in the preoperative and early postoperative period: a multivariate analysis in the midterm follow-up. Skull Base. 2007; 17:157-71. https://doi.org/10.1055/s-2007-970554.

6. Thompson LD, Gyure KA. Extracranial sinonasal tract meningiomas: a clinicopathologic study of 30 cases with a review of the literature. Am J Surg Pathol. 2000; 24: 640-50.

7. Maillo A, Orfao A, Sayagues JM, Diaz P, Gomez-Moreta JA, Caballero M, Santamarta D, Santos-Briz A, Morales F, Tabernero MD. New classification scheme for the prognostic stratification of meningioma on the basis of chromosome 14 abnormalities, patient age, and tumor histopathology. J Clin Oncol. 2003; 21:3285-95. https:// doi.org/10.1200/JCO.2003.07.156.

8. Derome PJ, Guiot G. Bone problems in meningiomas invading the base of the skull. Clin Neurosurg. 1978; 25:435-51.

9. Kasuya H, Kubo O, Tanaka M, Amano K, Kato K, Hori T. Clinical and radiological features related to the growth potential of meningioma. Neurosurg Rev. 2006; 29:293-6. https://doi.org/10.1007/s10143-006-0039-3.

10. van Alkemade H, de Leau M, Dieleman EM, Kardaun JW, van Os R, Vandertop WP, van Furth WR, Stalpers LJ.
Impaired survival and long-term neurological problems in benign meningioma. Neuro Oncol. 2012; 14:658-66. https://doi.org/10.1093/neuonc/nos13.

11. Obeid F, Al-Mefty O. Recurrence of olfactory groove meningiomas. Neurosurgery. 2003; 53:534-43.

12. Tsikoudas A, Martin-Hirsch DP. Olfactory groove meningiomas. Clin Otolaryngol Allied Sci. 1999; 24:507-9.

13. Ojeman RG. Supratentorial meningiomas: clinical features and surgical management. Neurosurgery. 1996; 1:873-90.

14. Turazzi S, Cristofori L, Gambin R, Bricolo A. The pterional approach for the microsurgical removal of olfactory groove meningiomas. Neurosurgery. 1999; 45:821-6.

15. Alaywan M, Sindou M. [Prognostic factors in the surgery for intracranial meningioma. Role of the tumoral size and arterial vascularization originating from the pia mater. Study of 150 cases]. [Article in French]. Neurochirurgie. 1993; 39:337-47.

16. Trittmacher S, Traupe H, Schmid A. Pre- and postoperative changes in brain tissue surrounding a meningioma. Neurosurgery. 1988; 22:882-5.

17. Philippon J, Foncin JF, Grob R, Srour A, Poisson M, Pertuiset BF. Cerebral edema associated with meningiomas: possible role of a secretory-excretory phenomenon. Neurosurgery. 1984; 14:295-301.

18. Ohnishi T, Posner JB, Shapiro WR. Vasogenic brain edema induced by arachidonic acid: role of extracellular arachidonic acid in blood-brain barrier dysfunction. Neurosurgery. 1992; 30:545-51.

19. Vaz R, Borges N, Cruz C, Azevedo I. Cerebral edema associated with meningiomas: the role of peritumoral brain tissue. J Neurooncol. 1998; 36:285-91.

20. Tanaka M, Imhof HG, Schucknecht B, Kollias S, Yonekawa Y, Valavanis A. Correlation between the efferent venous drainage of the tumor and peritumoral edema in intracranial meningiomas: superselective angiographic analysis of 25 cases. J Neurosurg. 2006; 104:382-8. https://doi. org/10.3171/jns.2006.104.3.382.

21. Yoshioka H, Hama S, Taniguchi E, Sugiyama K, Arita K, Kurisu K. Peritumoral brain edema associated with meningioma: influence of vascular endothelial growth factor expression and vascular blood supply. Cancer. 1999; 85:936-44.

22. Mathiesen T, Lindquist C, Kihlstrom L, Karlsson B. Recurrence of cranial base meningiomas. Neurosurgery. 1996; 39:2-9.

23. Domingues PH, Sousa P, Otero A, Goncalves JM, Ruiz L, de Oliveira C, Lopes MC, Orfao A, Tabernero MD. Proposal for a new risk stratification classification for meningioma based on patient age, WHO tumor grade, size, localization, and karyotype. Neuro Oncol. 2014; 16:735-47. https://doi. org/10.1093/neuonc/not325. 